\title{
$T$-IDEALS AND $c$-IDEALS
}

\author{
by AARON KLEIN \\ (Received 27th May 1977)
}

1.

Given a ring $R$ we consider the category $\tilde{R}$ of $R$-rings (rings $A$ with given ring-homomorphisms $R \rightarrow A$ ), and $R$-homomorphisms (ring-homomorphisms that form commutative triangles with the given maps from $R$ ). All rings are associative and have 1 , all homomorphisms send 1 to 1 .

We define a $c$-R -ring as an object $A$ in $\tilde{R}$ with a family of maps $\left\{\rho_{x} \in \operatorname{hom}_{\tilde{R}}(A, A) \mid x \in\right.$ $A\}$. Equivalently, a $c-R$-ring is an $R$-ring $A$ with a binary operation $a \cdot b\left(=a \rho_{b}\right)$ on $A$ satisfying

$$
\left(a+a^{\prime}\right) \cdot b=(a \cdot b)+\left(a^{\prime} \cdot b\right) ; \quad\left(a a^{\prime}\right) \cdot b=(a \cdot b)\left(a^{\prime} \cdot b\right) ; \hat{r} \cdot b=\hat{r}
$$

for all $a, a^{\prime}, b \in A, r \in R$. Here $\hat{r}$ denotes the image of $r$ under $R \rightarrow A$ and the ring multiplication on $A$ is denoted by juxtaposition.

We call the third operation $R$-composition and we denote by $c \tilde{R}$ the category whose objects are the $c-R$-rings and whose maps are those maps of $\tilde{R}$ which preserve $R$-composition.

Let $A$ be a $c$-R-ring and $K \subset A$. We call $K$ a $c$-ideal in $A$ if $K$ is the kernel of a map in $c \tilde{R}$. The following is implied.

Theorem 1. $K \subset A$ is a c-ideal in $A$ if and only if:

(i, $K$ is an ideal in the ring $A$,

(i, $k \cdot a \in K$ for all $k \in K, a \in A$, and

(i $\left.i_{3}\right) a \cdot\left(k+a^{\prime}\right)-a \cdot a^{\prime} \in K$ for all $a, a^{\prime} \in A, k \in K$.

2.

We emphasise the importance of $c$-ideals by relating them to the well-known $T$-ideals $(1,2.2)$ in free algebras.

Let $\Lambda$ be a commutative ring with 1 and $V=\Lambda\left\{x_{s}\right\}_{s \in S}$ the free associative $\Lambda$-algebra with 1 over a set $S$ (the notation follows (1)). Let $V^{|S|}$ be the direct product of $|S|$ copies of $V$. We may write elements of $V^{|S|}$ as vectors of polynomials in the (non-commuting) indeterminates $\left\{x_{s} \mid s \in S\right\}$, namely

$$
\mathbf{f}=\left(f_{s}(\mathbf{x})\right)_{s \in S} \text { with } \mathbf{x}=\left(x_{s}\right)_{s \in S},
$$

with component-wise addition and multiplication. We define a composition on $V^{|s|}$

$$
\mathbf{f} \circ \mathbf{g}=\left(f_{s}(\mathbf{g})\right)_{s \in S} \text { for } \mathbf{f}=\left(f_{s}(\mathbf{x})\right), \mathbf{g}=\left(g_{s}(\mathbf{x})\right) \text {. }
$$


It follows that $V^{|S|}$ with the (associative) composition (2) may be viewed as an object of the category $c \tilde{\Lambda}$.

Theorem 2. (i) If $K$ is a c-ideal in $V^{|S|}$ with the composition (2), then $K$ is a subdirect sum of $|S|$ copies of a $T$-ideal $J$ of the free algebra $V$ and it contains the direct sum $\Sigma_{|S|} J$ of $|S|$ copies of $J$; thus $K$ is a dense sub-direct sum.

(ii) For any $T$-ideal $J$ of the free algebra $V$, the direct product $J^{|S|}$ of $|S|$ copies of $J$ is a $c$-ideal in $V^{|S|}$. If the index-set $S$ is infinite and $J \neq 0$ then the direct sum $\Sigma_{|S|} J$ is not a $c$-ideal in $V^{|S|}$.

Proof. (i) Let $K$ be a $c$-ideal in $V^{|S|}$ and for any $r \in S$ denote by $J_{r}$ the $r$-th projection of $K$ into $V$. We show that $J_{r}$ is a $T$-ideal in the free algebra $V$. Take $h \in J_{r}$ and any $h \in K$ with $h_{r}=h$. For arbitrary $f \in V^{|S|}$ we have hof $\in K$ by $\left(i_{2}\right)$ of Theorem 1 , hence (hof), $\in J_{r}$. But $(h \circ f)_{r}=h_{r}(f)=h(f)$ and this proves that $J_{r}$ is indeed a $T$-ideal. We use the property $\left(i_{3}\right)$, Theorem 1, of $K$ to show that all $J_{r}$ are equal. Let $r, t$ be any two indices in $S$ and $h$ any element of $J_{r}$. Take again $h \in K$ with $h_{r}=h$ and $f=\left(\delta_{s t} x_{r}\right)_{s \in S}$. Then the vector $\mathbf{f \circ}(\mathbf{h}+O)-\mathbf{f \circ} O$ is in $K$ so its $t$-component, namely $f_{t}(\mathbf{h})=h$, is in $J_{t}$. This proves $J_{r} \subset J_{t}$ and, since $r$ and $t$ were arbitrary, it follows that all the projections of $K$ are equal, say to $J$. Now, the set of vectors $\left\{\left(\delta_{s t} h\right)_{s \in S} \mid t \in S, h \in J\right\}$ generates the direct sum $\Sigma_{|S|} J$. We show that all these vectors are in $K$, hence $\Sigma_{|S|} J \subset K$. Fix $t \in S$ and put $f=\left(\delta_{s t} x_{s}\right)_{s \in S}$. With any $h \in J$ take $h \in K$ with $h_{t}=h$. Then $\mathrm{f}(h+O)-\mathrm{f} \circ O$ is in $K$ and this vector is precisely $\left(\delta_{s t} h\right)_{s \in S}$.

(ii) Assume $J$ is a $T$-ideal in the free algebra $V$ and let $K$ be a direct product of $|S|$ copies of $J$. We show that $K$ is a $c$-ideal in $V^{|S|}$. The condition $\left(i_{1}\right)$ of Theorem 1 is evident and $\left(i_{2}\right)$ follows since $J$ is a $T$-ideal. To establish that $K$ meets $\left(i_{3}\right)$ we have to show that $f(h+g)-f(g) \in J$ for all $h \in K$ and for any $f \in V, g \in V^{|S|}$. It suffices to show it for monomials $f=\lambda x_{s_{1}} \ldots x_{s_{m}}$ in $V$. Thus we prove that $\lambda\left(h_{s_{1}}+g_{s_{1}}\right) \ldots\left(h_{s_{m}}+g_{s_{m}}\right)-\lambda g_{s_{1}} \ldots g_{s_{m}}$ is in $J$. Upon expanding, the term $g_{s_{1}} \ldots g_{s_{m}}$ cancels out and we arrive to a sum of monomials, each of them involving at least one component of $h$ as a factor. Since all the components of $h$ are in $J$ and $J$ is an ideal, the result follows.

To establish the last assertion in (ii), let $L=\Sigma_{|S|} J, J$ a non-zero $T$-ideal in $V$. Take any $h \in L$ with a certain non-zero component $h_{s_{0}}$. Now, consider $f \in V^{|S|}$ with all components equal to $x_{s_{0}}$. If $L$ satisfies $\left(i_{3}\right)$ of Theorem 1 , then $f \circ(h+O)-f \circ h$ has to be in $L$. Yet all the components of $\mathrm{fo}(\mathrm{h}+\mathrm{O})-\mathrm{f} \circ \mathrm{h}$ are equal to the non-zero polynomial $\boldsymbol{h}_{s_{0}}$, hence this vector cannot be in $L$ if $S$ is infinite. So, in this case $L$ cannot be a $c$-ideal.

Remark. The assumption that $V$ has 1 is not essential. It puts $V$ in $\bar{\Lambda}$, but the theorem is true even without 1 and the proof remains unaltered.

3.

For infinite $S$, the question whether there are $c$-ideals in $V^{|S|}$ which are not direct products of $T$-ideals, namely $J^{|S|}$, remains open. In this form, the problem is related to a well-known open problem (2) concerning varieties of algebras, as follows. We assume that $|S| \geqq|\Lambda|$, so $\left|\Lambda\left\{x_{s}\right\}_{s \in S}\right|=|S|$. 
Theorem 3. If there is in $V=\Lambda\left\{x_{s}\right\}_{s \in s} a T$-ideal $J$ which is not finitely generated as a $T$-ideal, then there is a c-ideal in $V^{|S|}$ which is not a direct product of $T$-ideals.

Proof. We may assume that $J$ is generated by a set of polynomials $\left\{p_{s} \mid s \in S\right\}$. For finite subsets $H \subset S$ denote by $J_{H}$ the $T$-ideal in $V$ generated by $\left\{p_{s} \mid s \in H\right\}$. Let $K_{H}$ be a direct product of $|S|$ copies of $J_{H}$. Then $K_{H}$ is a $c$-ideal by (ii) of Theorem 2 , and so we obtain a directed set of $c$-ideals $\left\{K_{H} \mid H\right.$ finite, $\left.H \subset S\right\}$ in $V^{|S|}$. It follows that $K=\cup K_{H}$ is a $c$-ideal in $V^{|S|}$. However, $K$ is a subdirect sum of $|S|$ copies of $J$, but not the whole $J^{|S|}$ since $\mathbf{p}=\left(p_{s}\right)_{s \in S} \notin K$.

\section{REFERENCES}

(1) C. ProcesI, Rings with polynomial identities (M. Dekker, N.Y., 1973).

(2) W. SpECHT, Gesetze in Ringen 1, Mat. Zeit. 52 (1950), 557-589.

DepartMEnT OF MATHEMATICS

BAR-ILAN UNIVERSITY

RAMAT GAN, ISRAEL 